\title{
Seasonal Patterns and Relationships among Coccidian Infestations, Measures of Oxidative Physiology, and Immune Function in Free-Living House Sparrows over an Annual Cycle
}

\begin{abstract}
Péter L. Pap ${ }^{1, *}$
Laura Pătraş ${ }^{2}$

Gergely Osváth ${ }^{3}$

Deborah M. Buehler ${ }^{4}$

Maaike A. Versteegh ${ }^{5}$

Alina Sesarman ${ }^{2}$

Manuela Banciu ${ }^{2}$

Csongor I. Vágási ${ }^{1}$

${ }^{1}$ MTA-DE "Lendület” Behavioural Ecology Research Group, Department of Evolutionary Zoology and Human Biology, University of Debrecen, Egyetem tér 1, H-4032 Debrecen, Hungary; and Evolutionary Ecology Group, Hungarian Department of Biology and Ecology, Babeş-Bolyai University, Clinicilor Street 5-7, RO-400006 Cluj Napoca, Romania; ${ }^{2}$ Department of Molecular Biology and Biotechnology, BabeşBolyai University, Clinicilor Street 5-7, RO-400006 Cluj Napoca, Romania; and Molecular Biology Centre, Institute for Interdisciplinary Research in Bio-Nano-Sciences, BabeşBolyai University, Treboniu Laurean Street 42, RO-400271 Cluj Napoca, Romania; ${ }^{3}$ Museum of Zoology, Babeş-Bolyai University, RO-400006 Cluj Napoca, Clinicilor Street 5-7, Romania; ${ }^{4}$ Office of the Dean, Faculty of Arts and Science, University of Toronto, Toronto, Ontario, Canada; ${ }^{5}$ Animal Ecology Group, Centre for Ecological and Evolutionary Studies, University of Groningen, Groningen, The Netherlands
\end{abstract}

Accepted 2/23/2015; Electronically Published 3/25/2015

\begin{abstract}
Temporal variation in oxidative physiology and its associated immune function may occur as a result of changes in parasite infection over the year. Evidence from field and laboratory studies suggests links between infection risk, oxidative stress, and the ability of animals to mount an immune response; however, the importance of parasites in mediating seasonal change in physiological makeup is still debated. Also, little is known about the temporal consistency of relationships among parasite infestation, markers of oxidative status and immune function in wild animals, and whether variation in oxidative
\end{abstract}

${ }^{*}$ Corresponding author; e-mail: peterlpap@gmail.com.

Physiological and Biochemical Zoology 88(4):395-405. 2015. (C) 2015 by The University of Chicago. All rights reserved. 1522-2152/2015/8804-4130\$15.00. DOI: $10.1086 / 681243$ measures can be viewed as a single integrated system. To address these questions, we sampled free-living house sparrows (Passer domesticus) every 2 mo over a complete year and measured infestation with coccidian parasites as well as nine traits that reflect condition, oxidative physiology, and immune function. We found significant seasonal variation in coccidian infestation and in seven out of nine condition and physiological variables over the year. However, we found little support for parasite-mediated change in condition, oxidative physiology, and immune functions in house sparrows. In accordance with this, we found no temporal consistency in relationships between the intensity of infestation and physiology. Among measures of oxidative physiology, antioxidants (measured as the total antioxidant capacity and the concentration of uric acid in the plasma) and oxidative damage (measured through the level of malondialdehyde in plasma) positively and consistently covaried over the year, while no such associations were found for the rest of traits (body mass, total glutathione, and leukocyte numbers). Our results show that natural levels of chronic coccidian infection have a limited effect on the seasonal change of physiological traits, suggesting that the variation of the latter is probably more affected by short-term disturbances, such as acute infection and/or season-specific stress stimuli.

Keywords: birds, heterophil to lymphocyte ratio, leukocyte count, oxidative status, parasites, seasonality.

\section{Introduction}

Parasites exert strong and varying pressure on the physiological makeup of hosts over the year, and seasonal differences in response may depend on the change in infection load and the magnitude of defense (Nelson et al. 2002; Møller et al. 2003; Hasselquist 2007; Martin et al. 2008). The abundance of parasites and the selection pressure they exert on hosts may fluctuate over the year because of changes in environmental conditions facilitating transmission. For example, this is typical for malaria, viruses, and some ecto- and endoparasites, such as ticks, mites, and worms (e.g., Randolph 1975; Pap et al. 2010a; Lachish et al. 2011; reviewed by Nelson et al. 2002; Altizer et al. 2006). Host immune defense and its associated oxidative physiology may evolve in response to selection pressures arising from parasites, and if this is the case, then we expect peaks in defense to mirror peaks in offense. 
Several studies have shown that components of the immune system may vary over the year; however, divergent changes were found between species, populations, and physiological traits, with no clear pattern of high and low responses in particular seasons (Møller et al. 2003; Buehler et al. 2008a, 2012; Martin et al. 2008; Pap et al. 2010b, 2014; Hegemann et al. 2012a, 2012b, 2013; Horrocks et al. 2012). Sex differences in reproductive physiology or parental investment may also affect immune function, the defense capacity of the antioxidant system, and/or the oxidative damage (Alonso-Alvarez et al. 2007; Metcalfe and Alonso-Alvarez 2013; Rubolini et al. 2012). This might explain the sex-specific seasonal variation in immune responsiveness (Pap et al. 2010c) and potentially influence the redox state in a sex-specific manner as well.

Immune function affects the antioxidant system and the redox state (Costantini and Møller 2009; Monaghan et al. 2009; Sorci and Faivre 2009). Therefore, it might be responsible for oxidative stress, that is, the upset balance between the production of pro-oxidants (reactive oxygen species [ROS]) and antioxidant defenses and repair mechanisms (Halliwell and Gutteridge 2007; Monaghan et al. 2009). Annual changes in parasitism and defense are therefore expected to be mirrored by measures of oxidative physiology. Changes in oxidative stress over the year can be related to excess production of ROS and/or the periodic overwhelming of the antioxidant system by parasites and the activated immune system (Schaefer et al. 2008; Costantini et al. 2009; Sorci and Faivre 2009; Cohen et al. 2010). The limited number of studies investigating oxidative physiology over the year have provided mixed evidence perhaps because of variation in methodologies to assess oxidative status, taxonomic differences, varying environmental conditions, or sampling that covered only a limited period of the year (Costantini et al. 2009; Norte et al. 2009; van de Crommenacker et al. 2011; Raja-aho et al. 2012; Rubolini et al. 2012; Pap et al. 2014).

Parasite infection and immune activation may affect different aspects of oxidative physiology, including measures of antioxidant system and oxidative damage (Halliwell and Gutteridge 2007; Costantini and Møller 2009; Sorci and Faivre 2009; Sepp et al. 2012a). Likewise, different measures of oxidative physiology may indicate similar or dissimilar aspects of infection, immune activation, and oxidative stress (Cohen and McGraw 2009; Costantini and Verhulst 2009; Monaghan et al. 2009; Hõrak and Cohen 2010; Metcalfe and Monaghan 2013). However, little is known about the consistency of relationships among levels of infection, immune response, and different measures of oxidative status in wild animals or about whether variation in measures of oxidative stress can be viewed as a single coherent system (for biomedical literature, see Dotan et al. 2004; Cohen and McGraw 2009; Monaghan et al. 2009). Examining how these varying traits covary may tell us something about constraints on the phenotypic flexibility and the extent to which they allow animals to adjust their physiology to diverse environmental conditions encountered over the year. Previous studies of parasite-induced immune activation have shown that individuals with high parasite loads tend to produce stronger immune responses and larger changes in oxidative state (i.e., Sorci and Faivre 2009). High loads of coccidian parasites have this effect on some measures of immune and oxidative physiology (Hõrak et al. 2004; Baeta et al. 2008; Pap et al. 2009, 2011; Sepp et al. 2012a). However, it remains unknown whether these patterns would hold over the year in wild conditions, since these studies were based on birds from the breeding season or from captive individuals.

In our previous study on captive house sparrows (Passer domesticus), we showed that none of 10 immunological and oxidative physiology indices covaried consistently with coccidian infestation over the year and that the sexes exhibited similar patterns (Pap et al. 2014). Thus, we found little support for a parasite-mediated change in immune functions and oxidative status. These results contrast with other studies on wild species, which showed significant sex-dependent change in immunological and oxidative state over the year (Pap et al. 2010c; van de Crommenacker et al. 2011; Hegemann et al. 2012b, 2013; Horrocks et al. 2012; Rubolini et al. 2012). However, most of these studies did not measure change in parasite pressure over the year (but see Horrocks et al. 2012), making it difficult to pinpoint a direct association between variation in physiological state and parasite infection. In our study on captive house sparrows, we showed that the number of coccidians varied little over the year, which was somewhat expected because of the limited change in transmission conditions of the infective oocysts in an environment of captivity (Pap et al. 2014). Our results on house sparrows indicated that circumstances related to the captive environment may affect parasite-induced changes in physiological response over the year because of unseasonal and chronic infection induced by conditions in captivity (see Buehler et al. 2008b; Sepp et al. 2010). Responses to coccidians and the difference in annual patterns between sexes can be masked in captive compared with wild-living birds as a result of stress or other factors (Buehler et al. 2008b; Kuhlman and Martin 2010). Therefore, field studies are needed to uncover the role of parasites in annual variation in immune function and associated oxidative status in wild-living birds, where the natural transmission of parasites and host's response are not altered by captive conditions.

In this study, we sampled free-living house sparrows once every 2 mo throughout an annual cycle and measured infection by the common unicellular endoparasite, the Isospora coccidians. On the same birds, we measured three components of the antioxidant system (total antioxidant capacity [TAC], concentration of uric acid [UA], and total glutathione [ $[\mathrm{GSH}]$ ) and the oxidative damage to membrane lipids (concentration of malondialdehyde $[\mathrm{MDA}])$. We also measured three immunological variables to characterize the immune system and to study its response to infection over the annual cycle (heterophils, lymphocytes, and total leukocytes), and we calculated the heterophil to lymphocyte (H/L) ratio. The leukocyte profile is a general assay of the health status, and the $\mathrm{H} / \mathrm{L}$ ratio is a general measure of stress (reviewed by Davis et al. 2008). Most of these variables of oxidative physiology, immune function, and stress index have been shown to be affected by coccidians in wild and captive birds (Hõrak et al. 2004; Pap et al. 2009, 2011; 
Sepp et al. 2012a; Bókony et al. 2014). Our study design allowed us to test the hypothesis that seasonal change in immune function and oxidative physiology are related to naturally occurring levels of parasite infection. We first examine how different indices change over the annual cycle. We then analyze whether the relationship between coccidians and measures of condition, oxidative physiology, and leukocyte number changes among sampling months. Finally, we analyze the consistency of covariation between measures of condition, oxidative physiology, and leukocyte number over the year and whether the strength of correlations differs between months with low and high parasite load. We hypothesize that individual differences in condition may be more clear-cut during times of the year when the threat of parasitism is the highest. Only under severe conditions might clear relationships become apparent and might physiological traits be correlated (Dotan et al. 2004). If parasites impose strong selection pressures on their hosts, then hosts should allocate limited resources to immune function and oxidative physiology mainly during the part of the year when the risk of parasites exploiting hosts is high.

\section{Material and Methods}

\section{Data Collection}

We captured adult house sparrows using mist nets across six different annual cycle stages over one annual cycle (see below) at a farm situated at Pinticu $\left(46^{\circ} 56^{\prime} \mathrm{N}, 24^{\circ} 32^{\prime} \mathrm{E}\right)$, Transylvania, central Romania. The birds were captured on the following dates: April 12, $2012(N=32)$; June 20, $2012(N=30)$; August 15, $2012(N=29)$; October 10, $2012(N=29)$; December 16, $2012(N=30)$; and February 15, $2013(N=30)$. The sex ratio within capture seasons was $\sim 1: 1$, and each individual bird was measured only once during the study period. Following capture, all birds were individually ringed, blood sampled, and weighed. We collected about $250 \mu \mathrm{L}$ of blood into heparinized capillary tubes within $30 \mathrm{~min}$ of capture, and immediately after sampling, we made blood smears by spreading a drop of blood on a microscope slide. The blood samples were transported in cooling bags to the laboratory for further processing within $8 \mathrm{~h}$. The birds were placed in a large outdoor aviary $(4 \mathrm{~m} \times 5 \mathrm{~m} \times 2.5 \mathrm{~m}$; length $\times$ width $\times$ height $)$ situated at the campus of Babeş-Bolyai University, Cluj-Napoca, for a total of $2 \mathrm{~d}$. Following this acclimatization period in the aviary, the house sparrows were placed in individual cages to quantify the level of coccidian infestation by measuring the rate of oocyst shedding over two additional days (for details, see Pap et al. 2009). Infestation intensity was expressed as number of oocysts per gram of feces, and the average of the $2 \mathrm{~d}$ was used in the analyses. Birds were fed ad lib. with a mixture of seeds containing ground corn, sunflower, wheat, and oats. Their food was also supplemented with two grated boiled eggs distributed equally among all birds on every even day. Six birds died in small cages for unknown reasons; therefore, the sample size slightly differed between analyses (see "Results"). Following feces collection, all house sparrows were released in good health condition in the population of origin. One may assume that capturing and introducing the breeding birds into the aviaries may cause the starvation and death of their nestlings. Because our house sparrow population is huge (estimated to more than 300 pairs), and supposing a random capture of birds using standard mist netting protocols, we assumed that the probability of catching both parents of a nest was rather low. If we caught only one member of the pair, then one parent could brood and feed the nestlings in the absence of its mate, as was shown in different studies on house sparrows. After release, birds usually start to feed their nestlings, reinstalling the biparental care. However, because male birds do not incubate, we admit that a few number of nests with eggs were abandoned, at least in those cases where the incubating females were captured.

\section{Measuring Oxidative Physiology and Immune Function}

Blood was centrifuged for $5 \mathrm{~min}$ at $6,200 \mathrm{~g}$ to separate the plasma and packed cell fractions. The samples were stored at $-20^{\circ} \mathrm{C}$ until further analyses. The packed cell fraction of the blood samples collected on December 16, 2012, accidentally thawed; therefore, the tGSH could not be measured for this month.

\section{Total Antioxidant Capacity}

TAC was measured according to the method described by Erel (2004), with slight modifications as per Sepp et al. (2010). This assay relies on the ability of nonenzymatic antioxidants (e.g., UA, vitamins, sulfhydryl groups of proteins, GSH) to decolorize the blue-green $\mathrm{ABTS}^{+}\left(2,2^{\prime}\right.$-azino-bis(3-ethylbenzothiazoline6-sulfonate)) to a degree proportional to their concentrations, which can be measured spectrophotometically at $660 \mathrm{~nm}$. Briefly, the first absorbance was measured after mixing $5 \mu \mathrm{L}$ of plasma with $200 \mu \mathrm{L}$ of $0.4 \mathrm{M}$ acetate buffer ( $\mathrm{pH}$ 5.8) as a sample blank, and the last absorbance was measured 20 min after incubating the previous mix with $20 \mu \mathrm{L}$ of $10 \mathrm{mM} \mathrm{ABTS}^{+}$(Sigma A1888) in $30 \mathrm{mM}$ acetate buffer ( $\mathrm{pH}$ 3.6). An antioxidant of known concentration (Trolox, Sigma 23,881-3) was used as a standard for the calculation of antioxidant levels in the samples. The assay results are expressed as $\mathrm{mM}$ Trolox equivalents. Repeatability within and between plates in a subset of samples was very high (within plates: intraclass correlation coefficient [ICC] $=0.91,95 \%$ confidence interval $[\mathrm{CI}]=0.82-1.00, F_{13,14}=21.7, P<0.001$; between plates: $\mathrm{ICC}=0.94,95 \% \mathrm{CI}=0.89-0.99, F_{13,28}=52.0$, $P<0.001)$.

\section{Uric Acid}

Plasma UA concentration was determined from $5 \mu \mathrm{L}$ of plasma using a spectrophotometric uricase/peroxidase method (Uric Acid liquicolor kit, Human, Wiesbaden, Germany). Results are given as $\mathrm{mg} / \mathrm{dL}$ plasma (see details and repeatability in Bókony et al. 2014).

\section{Total Glutathione}

GSH is the most important intracellular, endogenous, nonenzymatic antioxidant (Galván and Alonso-Álvarez 2008). 
The tGSH concentration was assayed by means of a commercial kit (Sigma-Aldrich, St. Louis) and according to Galván and Alonso-Álvarez (2008) and Hõrak et al. (2010), with the following modifications. After thawing on ice, the erythrocyte pellet was washed three times with phosphate-buffered saline and spun at $600 \mathrm{~g}$ for $10 \mathrm{~min}$ at $4^{\circ} \mathrm{C}$. After that, we weighed the pellets $( \pm 0.001 \mathrm{mg})$ and deproteinized with $5 \%$ 5-sulfosalicylic acid (SSA; 1:1 w/v). For example, a 100-mg pellet was diluted in $100 \mu \mathrm{L}$ of $5 \%$ SSA. This solution was vigorously vortexed, kept on ice for $10 \mathrm{~min}$, and then centrifuged at $10,000 \mathrm{~g}$ for $10 \mathrm{~min}$ at $4^{\circ} \mathrm{C}$ to remove the precipitated proteins. The supernatant $(5 \mu \mathrm{L})$ was transferred to another test tube, diluted 10-fold, and used subsequently for tGSH detection, according to the manufacturer's instructions. Total GSH determination is based on a kinetic assay in which nanomoles of GSH cause a continuous reduction of 5,5'-dithiobis(2-nitrobenzoic acid) to 5-thio-2-nitrobenzoic acid (TNB), and the GSH oxidized to GSSG is subsequently recycled by GSH reductase and reduced nicotinamide adenine dinucleotide phosphate (NADPH). The yellow color of the TNB product is proportional to the GSH concentration, and its absorbance was measured spectrophotometrically at $412 \mathrm{~nm}$ at 1-min intervals for $5 \mathrm{~min}$. The change in absorbance was compared with that of a standard curve $\left(R^{2}=0.99\right)$ generated by serial dilution of reduced GSH. Results are given in nmoles of $\mathrm{tGSH} / \mathrm{mg}$ of erythrocytes (for details and repeatability, see Bókony et al. 2014).

\section{Malondialdehyde}

MDA is a carbonyl compound that results from the peroxidative degeneration of membrane polyunsaturated fatty acids by ROS; thus, it is a widely used marker of oxidative stress (Del Rio et al. 2005). MDA concentration was determined from $10 \mu \mathrm{L}$ of plasma by high performance liquid chromatography (HPLC) on an HPLC SUPELCOSIL LC-18 column (5- $\mu \mathrm{m}$ particle size; Sigma-Aldrich), with ultraviolet detection at $254 \mathrm{~nm}$ (Jasco, UV-2075 Plus, Japan; Bókony et al. 2014). The mobile phase was $30 \mathrm{mM}$ monopotassium phosphate $\left(\mathrm{KH}_{2} \mathrm{PO}_{4}\right)$ methanol $(65: 35, \%, \mathrm{v} / \mathrm{v})$, and the flow rate was $0.5 \mathrm{~mL} / \mathrm{min}$. The retention time of MDA recorded was around $6 \mathrm{~min}$. MDA concentration in the sample was determined using a calibration curve $\left(R^{2}=0.99\right)$ of a series of standards generated by acidic hydrolysis of 1,1,3,3-tetraethoxypropane (TEP; Sigma-Aldrich). Results are given as $\mu \mathrm{g} / \mathrm{mL}$ plasma and are not corrected for the dilution factor (for details and repeatability, see Bókony et al. 2014).

\section{Leukocyte Count}

The blood smear was air dried, fixed in Dia-Fix, and stained with Dia-Red and Dia-Blue Panoptic (Diagon, Hungary). Smears were examined at $\times 1,000$ magnification, and the proportion of different types of leukocytes was assessed by counting 50 leukocytes. The number of white blood cells of different types was expressed per approximately 10,000 eryth- rocytes. We excluded monocytes, eosinophils, and basophils from the analyses because of their low concentration in the blood (less than 3 cells per 10,000 erythrocytes). Leukocytes were counted by the same person (L. Pătraş) and were moderately to highly repeatable (ICC $>0.45$ and $P<0.05$ in all cases).

\section{Statistical Analysis}

To examine whether and how different indices change over the annual cycle, we tested the effects of sampling month, sex, and the interaction between the two variables on condition, oxidative physiology, and leukocyte number separately for each dependent variable with general linear models (GLMs). To analyze whether the relationship (correlation) between coccidians and measures of condition, oxidative physiology, and leukocyte number changes among sampling months, we correlated coccidian infestation and variables of condition, oxidative physiology, and immune traits separately for the six sampling periods, using GLMs with sex included as a factor. Finally, to analyze the consistency of covariation between measures of condition, oxidative physiology, and leukocyte number over the year and whether the strength of correlations differs between months with low and high parasite load, we performed bivariate regressions between the different indices of condition, oxidative physiology, and immune traits during the six sampling periods, using GLMs with sex included as a factor.

Some of the plasma samples were hemolyzed, which might affect the measures of oxidative physiology. To control for the possible confounding effect of hemolysis, we visually scored plasma samples between 0 and 3, where the lowest value means the absence of hemolysis. TAC, UA, and MDA were affected by the level of hemolysis; therefore, we included the scores as factors in the models. However, for perspicuity, we do not show the effect of hemolysis. We used $\log _{10}(x+1)$ transformation on the number of oocysts per gram feces, UA, heterophils, lymphocytes, H/L ratio, and total leukocytes. These transformations resulted in normally distributed residuals. All other variables conformed to normality of residuals without transformation.

\section{Results}

Coccidian infestation, body mass, measures of oxidative physiology, and leukocyte count-but not $\mathrm{H} / \mathrm{L}$ ratio or $\mathrm{MDA}-$ varied significantly over the year (fig. 1; table 1); however, the pattern depended on the measure. Coccidian infestation was highest during June and lowest during August and the winter periods (December to February). All measures, except those pertaining to leukocyte numbers, were similar between the sexes, as revealed by the nonsignificant effects of the sex and sex $\times$ season interaction (fig. 1; table 1 ). The numbers of heterophils, lymphocytes, and total leukocytes were higher in females than in males during the breeding period (from April 

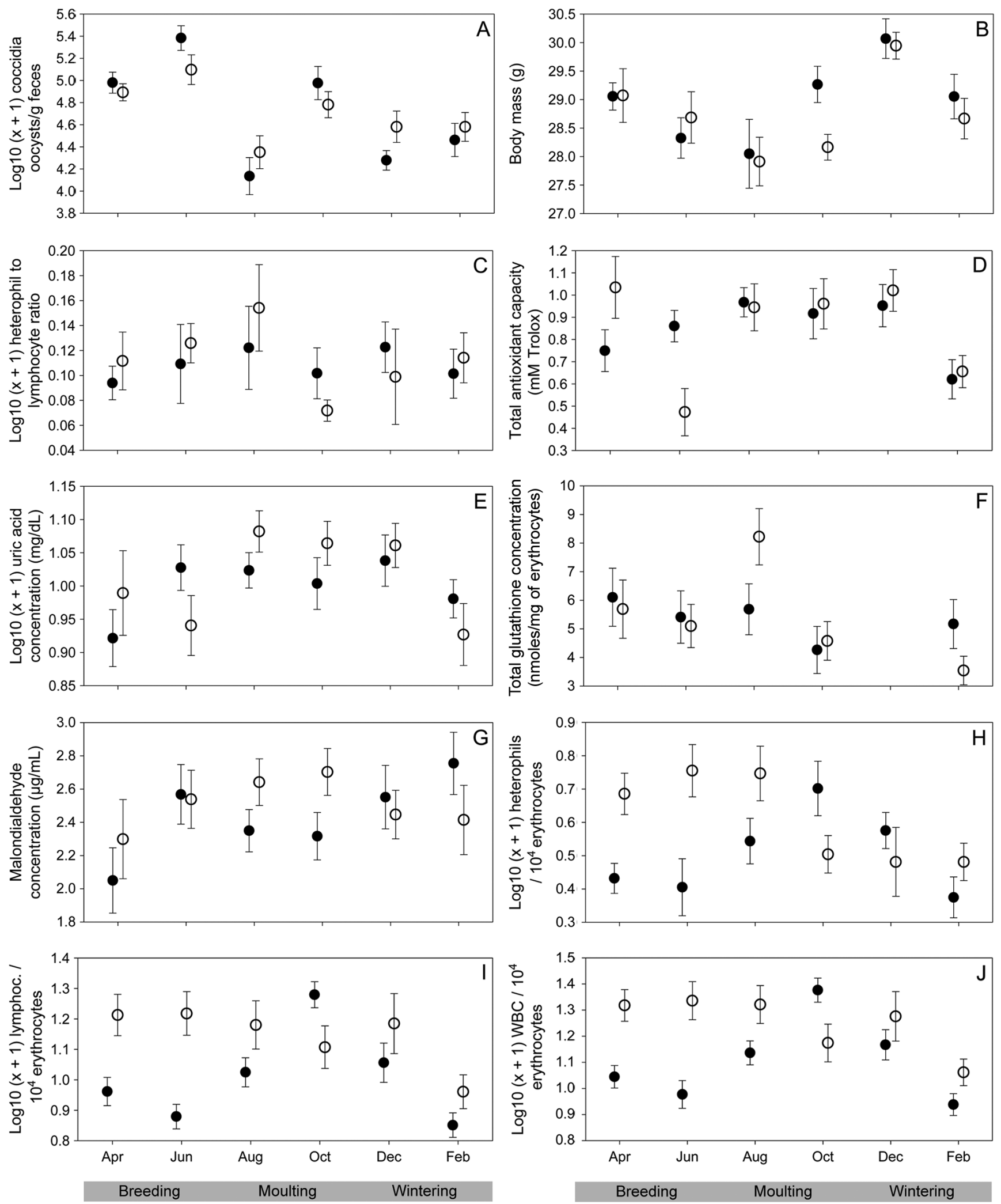

Figure 1. Seasonal variation of coccidian infestation $(A)$, condition and stress $(B, C)$, antioxidant capacity and oxidative state $(D-G)$, and leukocyte numbers $(H-J)$ in house sparrows. Open and filled circles indicate females and males, respectively. Means $\pm 1 \mathrm{SE}$ error bars are plotted. 
Table 1: Results of the general linear models (GLMs) on the effect of sampling month and sex on coccidian infestation, body mass, oxidative stress, and leukocyte number in house sparrows

\begin{tabular}{|c|c|c|c|c|c|c|c|c|c|}
\hline \multirow[b]{2}{*}{ Variable } & \multicolumn{3}{|c|}{ Season } & \multicolumn{3}{|c|}{ Sex } & \multicolumn{3}{|c|}{ Season $\times$ sex } \\
\hline & $\mathrm{df}$ & $F$ & $P$ & df & $F$ & $P$ & df & $F$ & $P$ \\
\hline \multicolumn{10}{|l|}{ Pathogen load: } \\
\hline Coccidian infestation & 5,160 & 17.1 & $<.001$ & 1,160 & .03 & .87 & 5,160 & 1.7 & .14 \\
\hline \multicolumn{10}{|l|}{ Condition and stress: } \\
\hline Body mass & 5,168 & 6.2 & $<.001$ & 1,168 & 1.1 & .30 & 5,168 & .8 & .53 \\
\hline Heterophil to lymphocyte ratio & 5,167 & 1.0 & .45 & 1,167 & .1 & .76 & 5,167 & .5 & .76 \\
\hline \multicolumn{10}{|l|}{ Oxidative state: } \\
\hline Total antioxidant capacity & 5,158 & 6.0 & $<.001$ & 1,158 & .0 & .98 & 5,158 & 2.1 & .08 \\
\hline Uric acid & 5,163 & 2.5 & .03 & 1,163 & .3 & .62 & 5,163 & 1.3 & .25 \\
\hline Total glutathione & 4,137 & 3.1 & .02 & 1,137 & .03 & .86 & 4,137 & 1.6 & .19 \\
\hline Malondialdehyde & 5,163 & 1.2 & .30 & 1,163 & .5 & .50 & 5,163 & 1.0 & .41 \\
\hline \multicolumn{10}{|l|}{ Leukocyte number: } \\
\hline Heterophils & 5,167 & 2.3 & .06 & 1,167 & 6.5 & .01 & 5,167 & 4.5 & $<.001$ \\
\hline Lymphocytes & 5,167 & 4.9 & $<.001$ & 1,167 & 14.4 & $<.001$ & 5,167 & 3.9 & $<.01$ \\
\hline White blood cells & 5,167 & 5.1 & $<.001$ & 1,167 & 16.5 & $<.001$ & 5,167 & 5.1 & $<.001$ \\
\hline
\end{tabular}

Note. In the case of total antioxidant capacity, uric acid, and malondialdehyde, the degree of hemolysis of the plasma was included in the models to control for its effect. Values in bold indicate statistical significance.

to August) but differed little between the sexes during the rest of the year (October, December, and February).

We used bivariate correlations - while controlling for the potentially confounding effect of the hemolysis score of the plasma in the case of TAC, UA, and MDA (see "Material and Methods") and sex-to examine the consistency of interseasonal correlations at the month level. The ellipse plots clearly show that covariations are inconsistent, and correlations between coccidian infestation and condition indices, measures of oxidative physiology, and leukocyte counts over the annual cycle are generally low (statistically nonsignificant; fig. 2). There was no significant correlation between coccidians and measures of physiology even in June, when the infestation of the birds was at its peak.

Using the same bivariate analyses, we examined the consistency of interseasonal correlations at the month level between condition indices, measures of oxidative physiology, and leukocyte counts. The ellipse plots show that most of the correlations between different measures vary inconsistently over the year (fig. 3), and correlations do not appear to be related to coccidian infestation, since the lack of correlation remained in June when the coccidian infestation was the highest as well as during the rest of the year (fig. 3). However, consistent correlations over the year emerged between some measures, for instance, the number of heterophil and lymphocyte leukocytes and the derived $\mathrm{H} / \mathrm{L}$ ratio stress index. We also found consistent and strong associations between TAC, $\mathrm{UA}$, and MDA; all were significantly and positively correlated with each other (fig. 3). The strength of correlations between measures of oxidative physiology and leukocyte count were generally low, and the direction of association changed over the year (fig. 3).

\section{Discussion}

Seasonal Change of Coccidians, Condition, and Physiological Traits

We found significant annual variation in coccidian infestation and in seven of nine condition, oxidative physiology, and immune traits over the year. Annual variation in the number of coccidian oocysts shed showed a marked difference to our previous study on captive house sparrows (Pap et al. 2014), indicating that captive conditions may significantly alter the transmission and seasonal shedding of coccidians in this species. In our study on house sparrows living in aviaries, we found that coccidian infestation varied little over the year. This may indicate that transmission rates in captivity do not fluctuate over the year as they might in the wild and/or that the relaxed nutritional and energetic constraints on the captive birds might also reduce the fluctuations in the number of coccidian oocysts shed. Although the number of oocysts shed varies more in the wild than in captivity (see Pap et al. 2014), we found little support for parasite-mediated change in oxidative physiology and immune functions in free-living house sparrows (in contrast to van de Crommenacker et al. 2012; reviewed in Hasselquist 2007; Martin et al. 2008). This was supported by the absence of consistent correlation between the number of coccidians and the measured physiological traits between months (fig. 2). Thus, we found no evidence that coccidians mediate changes in physiological traits in either our earlier study on captive house sparrows or this study on free-living house sparrows. Our results suggest that factors other than coccidians affect the physiological makeup of the birds. In freeliving birds, such other factors might include breeding effort, hormonal state, and stress due to infection and nutritional 


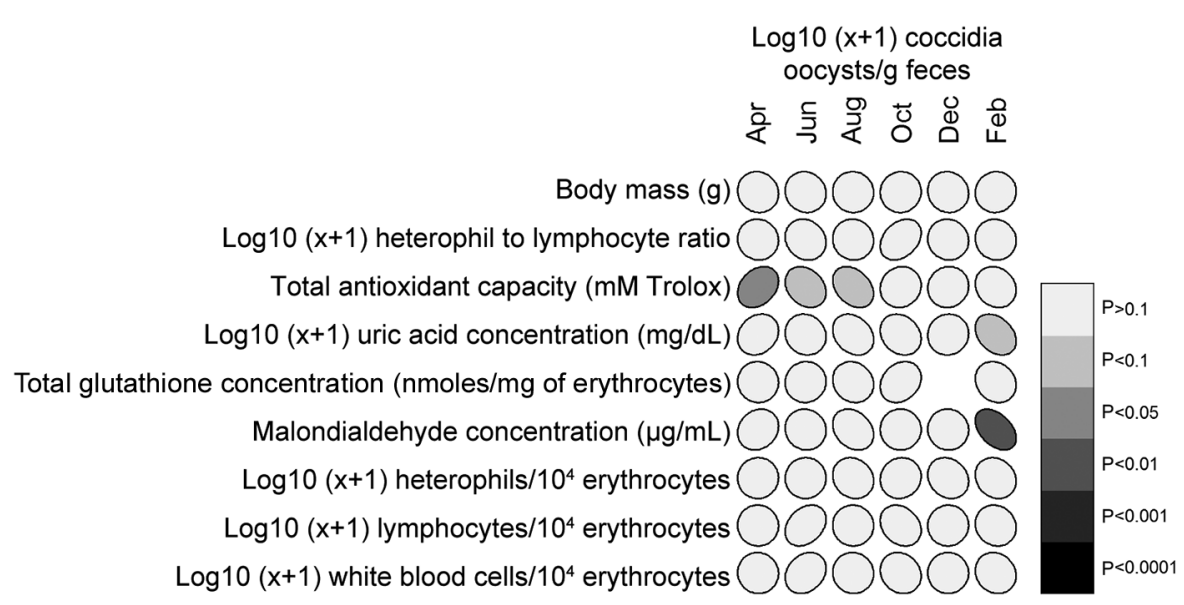

Figure 2. Correlations between coccidian infestation (columns) and condition indices, measures of oxidative physiology, and leukocyte number (rows) over an annual cycle in house sparrows. Each ellipse represents a correlation within a given month, where the effect of sex was controlled in the models. The ellipse for total glutathione in December is missing because of absence of data. Right-tilted ellipses indicate positive correlations, and left-tilted ellipses indicate negative correlations. The narrowness of ellipses is proportional to the strength of correlation ( $r$ values), and the shading is proportional to the significance level ( $P$ values).

status (Alonso-Alvarez et al. 2007; Schaefer et al. 2008; Mougeot et al. 2009; Noguera et al. 2011; van de Crommenacker et al. 2011; Rubolini et al. 2012). In captive birds, other factors might include suppression of breeding, a continuous supply and uniform composition of food over the year, and stress due to captivity (Buehler et al. 2008b; Kuhlman and Martin 2010; Sepp et al. 2010).

We found that measures of the antioxidant system (TAC, $\mathrm{UA}$, and $\mathrm{tGSH}$ ) varied inconsistently over the year, which may explain the absence of seasonal variation in oxidative damage (measured as the concentration of MDA). We speculate that the absence of a clear seasonal pattern in measures of oxidative physiology can be related to the continuous finetuning of oxidative status in order to maintain optimal balance between the production of pro-oxidants and the antioxidant system (Costantini and Verhulst 2009; Monaghan et al. 2009; Hõrak and Cohen 2010; Metcalfe and Monaghan 2013). The absence of differences between sexes in any measures of oxidative physiology is in line with this hypothesis.

We found significant sex differences in the number of leukocytes in wild-living house sparrows, while males and females of captive birds were more similar over the year (see Pap et al. 2014). The higher concentration of heterophils, lymphocytes, and total leukocytes in free-living females during the breeding period (from April to August; fig. $1 H-1 J$ ) suggests that during this period of the year, males are immunologically suppressed and/or the defense system of females is upregulated (Møller et al. 2003; Hasselquist 2007; Martin et al. 2008). Other measures of immune function in this species support the stronger immune function in female than in male free-living birds during the mating season (Pap et al. 2010c). During the mating and the breeding season, males are engaged in costly sexual behavior (Anderson 2006), which may explain their reduced immune function related to females at this period of the year. Outside of the reproductive period, sexes are more similar in their behavioral and hormonal status, which is reflected in the similarity in the number of leukocytes (this study) and other immune traits (Pap et al. 2010c) between males and females. Similarly, captive birds were prevented from breeding, reducing sex-specific behavioral and hormonal differences, which may explain the absence of differences in immune function between sexes in captivity (Pap et al. 2014).

In contrast to patterns in leukocyte numbers, we found little support for sexual dimorphism in oxidative physiology in freeliving house sparrows, despite the putative effects of parasites, immune activation, and other factors (i.e., hormones) on oxidative status via several mutually nonexclusive physiological pathways (von Schantz et al. 1999; Alonso-Alvarez et al. 2007; Galván and Alonso-Álvarez 2008; Mougeot et al. 2009). Our results are in contrast to other studies, where differences between males and females in measures of oxidative physiology were found (Costantini et al. 2009; Norte et al. 2009; van de Crommenacker et al. 2011; Raja-aho et al. 2012; Rubolini et al. 2012). We suggest that the similarity in measures of oxidative physiology between male and female house sparrows may be related to the continuous fine-tuning of oxidative status in order to maintain optimal balance between the production of pro-oxidants and the antioxidant system (Costantini and Verhulst 2009; Monaghan et al. 2009; Hõrak and Cohen 2010; Metcalfe and Monaghan 2013).

\section{The Effect of Coccidians on Condition and Physiological Measures over the Year}

The absence of an effect of chronic coccidian infestation on physiological measures is somewhat surprising because, unlike in captive birds, in free-living birds the number of coccidians changed markedly over the year. Generally, house sparrows and other free-living birds are negatively affected by coccid- 


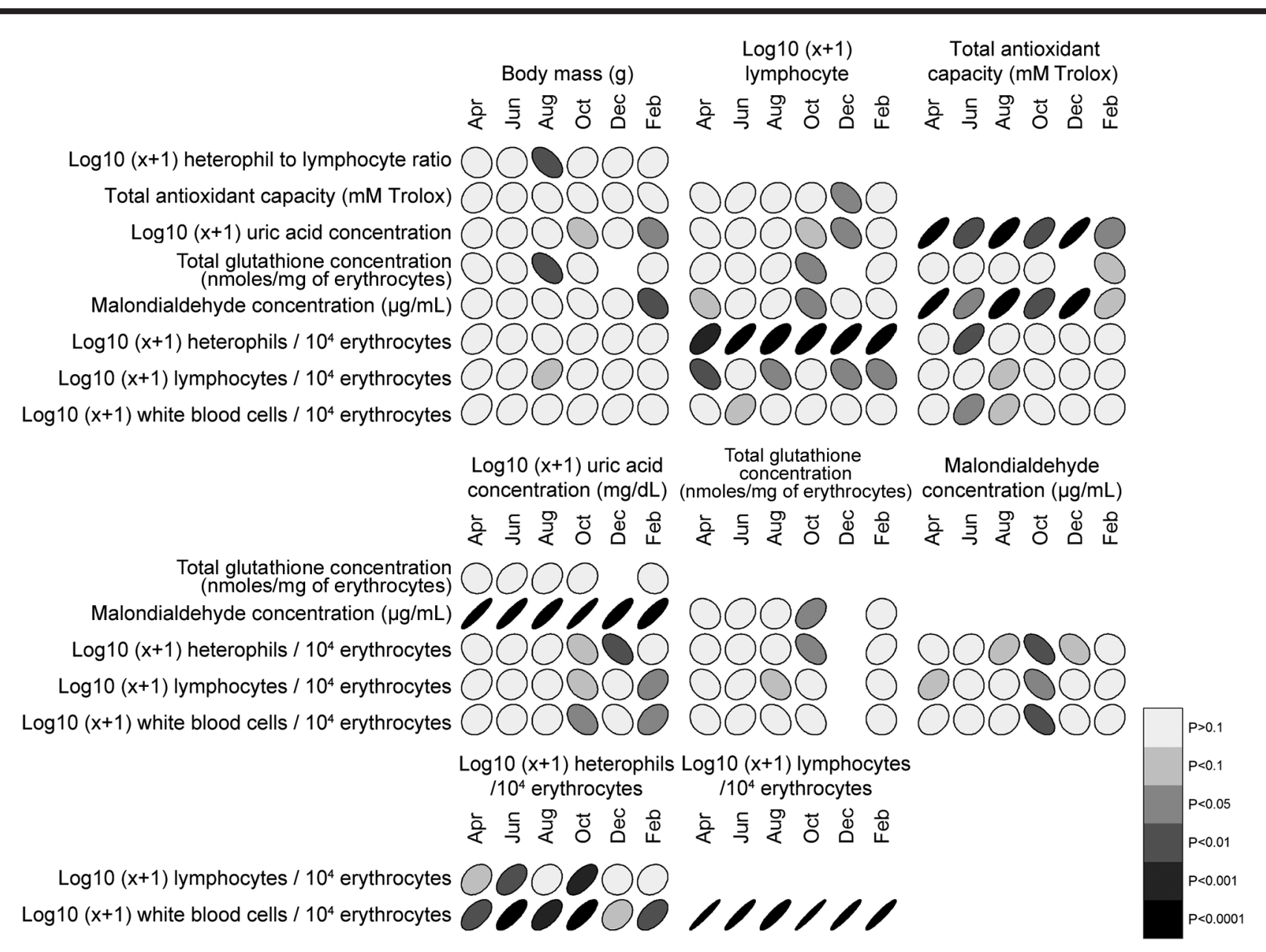

Figure 3. Correlations between condition indices, measures of oxidative physiology, and leukocyte number over an annual cycle in house sparrows. Each ellipse represents a correlation within a given month, where the effect of sex was controlled in the models. The ellipses for total glutathione in December are missing because of absence of data. Right-tilted ellipses indicate positive correlations, and left-tilted ellipses indicate negative correlations. The narrowness of ellipses is proportional to the strength of correlation ( $r$ values), and the shading is proportional to the significance level ( $P$ values).

ians in terms of condition, measures of oxidative physiology, and immune function (Hõrak et al. 2004; Pap et al. 2009, 2011; Sepp et al. 2012a; Bókony et al. 2014). However, most of these studies were conducted on birds with acute (experimental) infestation, which may explain the differences between this study and other studies. Our results suggest that hosts may physiologically adjust to chronic coccidian infestation; however, this may not prevent the reduction of fitness over prolonged exposure to these parasites (see Pap et al. 2013). For example, traits that are directly connected to nutritional supply (e.g., feather growth) are affected more by the nutritional diversion caused by these parasites (Hõrak et al. 2004; Baeta et al. 2008; Pap et al. 2009, 2013).

\section{Covariation of Condition and Physiological Traits}

We found limited support for the idea that covariation between measures of condition, oxidative physiology, and leukocyte count is constrained, as indicated by the absence of consistent covariation among these traits over the year (except TAC, UA, and MDA; fig. 3). TAC and UA were highly and positively correlated, indicating that these measures can be viewed as a single and coherent system (Cohen and McGraw 2009; Sepp et al. 2012b). Also, MDA concentration was positively associated with TAC and UA, indicating that an increase in oxidative damage is consistently (and independently of season) followed by an increase in extracellular, nonenzymatic antioxidants. These results strongly suggest that an increase in oxidative damage is a good proxy of the amount of antioxidants circulating in the blood, which can be explained by the need to reduce the amount of peroxidation on membrane lipids. However, because we cannot infer the causality in our correlations between different oxidative markers, our conclusions about subtle relationships between antioxidants and MDA remain to be demonstrated experimentally. On the other hand, none of the measures of oxidative physiology were consistently or tightly correlated with condition and leukocyte number, suggesting that the oxidative status is not constrained at 
any time of the year by the condition and health status of the individuals. Note, however, that all variables that resulted from differential and total leukocyte counts are nonindependent and related to each other, which may affect the relationship between physiological traits.

These results highlight the importance of measuring many aspects of the redox system, because the correlation between measures of antioxidants and oxidative damage to cellular macromolecules is sometimes weak and inconsistent between periods of the year (i.e., between MDA and TAC and between tGSH and MDA). Therefore, even if aspects of antioxidants and oxidative damage are all measured, conclusions will depend on which index is used to measure them. Our results strongly support Cohen and McGraw's (2009) comparative study in which a loose and evolutionarily flexible connection was found between different measures of antioxidants. Our study adds to their conclusion in the sense that we have shown that measures of oxidative physiology vary between different periods of the year within the same species, and this change seems significant above and beyond the marked interspecific changes they found. This finding may explain the contrasting results between studies on covariation of measures of oxidative physiology in different species (i.e., Simons et al. 2012) and supports what has been found for other measures of physiology, such as the metabolic rate, stress, and immune function (Buehler et al. 2011, 2012; Versteegh et al. 2012).

We hypothesized that individual differences in condition might be more clear-cut during times of the year when threats of parasitism are highest. Therefore, we predicted that at peak infestation, correlations between coccidian abundance and measures of oxidative stress and immune function would be most apparent. We further predicted that measures of oxidative stress and immune function would correlate with each other if constrained (Dotan et al. 2004). Our results do not support our expectations. Coccidian infestation in house sparrows tended to be highest during June and lowest during August and the winter periods (December to February). However, contrary to our predictions, the strength of correlation was low or nonsignificant independently of these contrasting periods, supporting our previous conclusion about the absence or limited effect of coccidians on seasonal variation in physiological traits. Furthermore, we found no consistent correlations between different measures of condition, oxidative physiology, and leukocyte count in any part of the year. During demanding periods of the annual cycle, such as reproduction and wintering, metabolic rates are normally higher (Daan et al. 1990; Broggi et al. 2007), potentially leading to higher generation of pro-oxidants and thus higher levels of oxidative damage, unless defense mechanisms by antioxidants and other means (e.g., uncoupling proteins) are sufficiently upregulated. However, comparing these periods with the rest of the sampling months did not reveal any consistent correlation between the traits measured, except TAC, UA, and MDA. These results strongly suggest independent and flexible responses of measures of oxidative physiology to changes in condition and leukocyte number.
In conclusion, this study shows that chronic coccidian infestation varies significantly over the year in wild-living house sparrows; however, this barely affects any of the nine measures of condition, oxidative physiology, and leukocyte count. The patterns of variation between parasite infestation and physiological measures did not mirror each other, contrary to what was predicted, nor did we find significant or consistent correlations between coccidian infestation and these traits over the year. Among the oxidative measures, we found consistent and positive correlations between TAC, UA, and MDA over the year; however, patterns of covariation were more flexible for the rest of the traits (low or no consistent bivariate correlations). Our results indicate that measures of condition, oxidative physiology, and leukocyte count are generally loosely integrated and that the direction of correlation varies markedly over the year. These loose associations between traits make it difficult to draw general conclusions about oxidative stress from a limited number of oxidative measures.

\section{Acknowledgments}

B. Bakó, Á. Péter, K. Sándor, M. Csiszár, A. Marton, and L. Bărbos kindly helped us during the fieldwork or measurement sessions. Elin Sild, José Carlos Noguera, Jonathan Blount, Adela Pintea, and Dumitrița Rugina kindly provided help with malondialdehyde, total glutathione, total antioxidant capacity, and uric acid assays. Two anonymous reviewers provided constructive comments on the manuscript. The research of P.L.P. was supported by TÁMOP-4.2.4.A/2-11/1-2012-0001 National Excellence Program. This project was sponsored by the European Union and the State of Hungary, cofinanced by the European Social Fund. C.I.V. was supported by a postdoctoral grant (GTC 34062/2013) of the Babeş-Bolyai University. The research infrastructure was supported by a research grant (PN II. RU TE 291/2010) of the Romanian Ministry of Education and Research. The experimental procedures were licensed by the Romanian Academy of Sciences (license 2257).

\section{Literature Cited}

Alonso-Alvarez C., S. Bertrand, B. Faivre, O. Chastel, and G. Sorci. 2007. Testosterone and oxidative stress: the oxidation handicap hypothesis. Proc R Soc B 274:819-825.

Altizer S., A. Dobson, P. Hosseini, P. Hudson, M. Pascual, and P. Rohani. 2006. Seasonality and the dynamics of infectious diseases. Ecol Lett 9:467-484.

Anderson T. 2006. Biology of the ubiquitous house sparrow: from genes to populations. Oxford University Press, Oxford.

Baeta R., B. Faivre, S. Motreuil, M. Gaillard, and J. Moreau. 2008. Carotenoid trade-off between parasitic resistance and sexual display: an experimental study in the blackbird (Turdus merula). Proc R Soc B 275:427-434.

Bókony V., Á.Z. Lendvai, C.I. Vágási, L. Pătraş, P.L. Pap, J. Németh, E. Vincze, et al. 2014. Necessity or capacity? physiological state predicts problem-solving performance in house sparrows. Behav Ecol 25:124-135. 
Broggi J., E. Hohtola, K. Koivula, M. Orell, R.L. Thomson, and J.-Å. Nilsson. 2007. Sources of variation in winter basal metabolic rate in the great tit. Funct Ecol 21:528-533.

Buehler D.M., T. Piersma, K.D. Matson, and B.I. Tieleman. 2008a. Seasonal redistribution of immune function in a shorebird: annual cycle effects override adjustments to thermal regime. Am Nat 172:783-796.

Buehler D.M., T. Piersma, and B.I. Tieleman. 2008b. Captive and free-living red knots Calidris canutus exhibit differences in non-induced immunity that suggest different immune strategies in different environments. J Avian Biol 39:560566.

Buehler D.M., M.A. Versteegh, K.D. Matson, and B.I. Tieleman. 2011. One problem, many solutions: simple statistical approaches help unravel the complexity of the immune system in an ecological context. PLoS ONE 6:e18592.

Buehler D.M., F. Vezina, W. Goymann, I. Schwabl, M.A. Versteegh, B.I. Tieleman, and T. Piersma. 2012. Independence among four physiological traits suggests flexibility in the face of ecological demands on phenotypes. J Evol Biol 25:1360-1613.

Cohen A.A., J.P. de Magalhães, and K. Gohil. 2010. Ecological, biomedical and epidemiological approaches to understanding oxidative balance and ageing: what they can teach each other. Funct Ecol 24:997-1006.

Cohen A.A. and K.J. McGraw. 2009. No simple measures for antioxidant status in birds: complexity in inter- and intraspecific correlations among circulating antioxidant types. Funct Ecol 23:310-320.

Costantini D., G. Dell'Omo, S.P. De Filippis, C. Marquez, H.L. Snell, H.M. Snell, W. Tapia, G. Brambilla, and G. Gentile. 2009. Temporal and spatial covariation of gender and oxidative stress in the Galápagos land iguana Conolophus subcristatus. Physiol Biochem Zool 82:430-437.

Costantini D. and A.P. Møller. 2009. Does immune response cause oxidative stress in birds? a meta-analysis. Comp Biochem Physiol A 153:339-344.

Costantini D. and S. Verhulst. 2009. Does high antioxidant capacity indicate low oxidative stress? Funct Ecol 23:506509.

Daan S., D. Masman, and A. Groenewold. 1990. Avian basal metabolic rates: their association with body composition and energy expenditure in nature. Am J Physiol 259:333-340.

Davis A.K., D.L. Maney, and J.C. Maerz. 2008. The use of leukocyte profiles to measure stress in vertebrates: a review for ecologists. Funct Ecol 22:760-772.

Del Rio D., A.J. Stewart, and N. Pellegrini. 2005. A review of recent studies on malondialdehyde as toxic molecule and biological marker of oxidative stress. Nutr Metab Cardiovasc Dis 15:316-328.

Dotan Y., D. Lichtenberg, and I. Pinchuk. 2004. Lipid peroxidation cannot be used as a universal criterion of oxidative stress. Prog Lipid Res 43:200-227.

Erel O. 2004. A novel automated direct measurement method for total antioxidant capacity using a new generation, more stable ABTS radical cation. Clin Biochem 37:277-285.
Galván I. and C. Alonso-Álvarez. 2008. An intracellular antioxidant determines the expression of a melanin-based signal in a bird. PLoS ONE 3:e3335.

Halliwell B. and J. Gutteridge. 2007. Free radicals in biology and medicine. Oxford University Press, Oxford.

Hasselquist D. 2007. Comparative immunoecology in birds: hypotheses and tests. J Ornithol 148:571-582.

Hegemann A., K.D. Matson, C. Both, and B.I. Tieleman. $2012 b$. Immune function in a free-living bird varies over the annual cycle, but seasonal patterns differ between years. Oecologia 170:605-618.

Hegemann A., K.D. Matson, M.A. Versteegh, and B.I. Tieleman. 2012a. Wild skylarks seasonally modulate energy budgets but maintain energetically costly inflammatory immune responses throughout the annual cycle. PLoS ONE 7:e36358.

Hegemann A., K.D. Matson, M.A. Versteegh, A. Villegas, and B.I. Tieleman. 2013. Immune response to an endotoxin challenge involves multiple immune parameters and is consistent among the annual-cycle stages of a free-living temperate zone bird. J Exp Biol 216:2573-2580.

Hõrak P. and A. Cohen. 2010. How to measure oxidative stress in an ecological context: methodological and statistical issues. Funct Ecol 24:2225-2233.

Hõrak P., L. Saks, U. Karu, I. Ots, P.F. Surai, and K.J. McGraw. 2004. How coccidian parasites affect health and appearance of greenfinches. J Anim Ecol 73:935-947.

Hõrak P., E. Sild, U. Soomets, T. Sepp, and K. Kilk. 2010. Oxidative stress and information content of black and yellow plumage coloration: an experiment with greenfinches. J Exp Biol 213:2225-2233.

Horrocks N.P.C., K.D. Matson, M. Shobrak, J.M. Tinbergen, and B.I. Tieleman. 2012. Seasonal patterns in immune indices reflect microbial loads on birds but not microbes in the wider environment. Ecosphere 3:19.

Kuhlman J.R. and L.B. Martin II. 2010. Captivity affects immune redistribution to skin in a wild bird. Funct Ecol 24: 830-837.

Lachish S., S.C.L. Knowles, R. Alves, M.J. Wood, and B.C. Sheldon. 2011. Infection dynamics of endemic malaria in a wild bird population: parasite species-dependent drivers of spatial and temporal variation in transmission rates. J Anim Ecol 80:1207-1216.

Martin L.B., II, Z.M. Weil, and R.J. Nelson. 2008. Seasonal changes in vertebrate immune activity: mediation by physiological trade-offs. Philos Trans R Soc B 363:321-339.

Metcalfe N.B. and C. Alonso-Alvarez. 2013. Oxidative stress as a life-history constraint: the role of reactive oxygen species in shaping phenotypes from conception to death. Funct Ecol 24:984-996.

Metcalfe N.B. and P. Monaghan. 2013. Does reproduction cause oxidative stress? an open question. Trends Ecol Evol 28:347-350.

Møller A.P., J. Erritzøe, and N. Saino. 2003. Seasonal changes in immune response and parasite impact on hosts. Am Nat 161:657-671. 
Monaghan P., N.B. Metcalfe, and R. Torres. 2009. Oxidative stress as a mediator of life history trade-offs: mechanisms, measurements and interpretation. Ecol Lett 12:75-92.

Mougeot F., J. Martínez-Padilla, L.M.I. Webster, J.D. Blount, L. Pérez-Rodríguez, and S.B. Piertney. 2009. Honest sexual signalling mediated by parasite and testosterone effects on oxidative balance. Proc R Soc B 276:1093-1100.

Nelson R.J., G.E. Demas, S.L. Klein, and L.J. Kriegsfeld. 2002. Seasonal patterns of stress, immune function, and disease. Cambridge University Press, Cambridge.

Noguera J.C., M. Lores, C. Alonso-Álvarez, and A. Velando. 2011. Thrifty development: early life diet restriction reduces oxidative damage during later growth. Funct Ecol 25:11441153.

Norte A.C., J.A. Ramos, J.P. Sousa, and B.C. Sheldon. 2009. Variation of adult great tit Parus major body condition and blood parameters in relation to sex, age, year and season. J Ornithol 150:651-660.

Pap P.L., G.Á. Czirják, C.I. Vágási, Z. Barta, and D. Hasselquist. 2010c. Sexual dimorphism in immune function changes during the annual cycle in the house sparrows. Naturwissenschaften 97:891-901.

Pap P.L., A. Sesarman, C.I. Vágási, D.M. Buehler, L. Pătraş, M.A. Versteegh, and M. Banciu. 2014. No evidence for parasitism-linked changes in immune function or oxidative physiology over the annual cycle of an avian species. Physiol Biochem Zool 87:729-739.

Pap P.L., C.I. Vágási, L. Bărbos, and A. Marton. 2013. Chronic coccidian infestation compromises flight feather quality in house sparrows Passer domesticus. Biol J Linn Soc 108:414428.

Pap P.L., C.I. Vágási, G.Á. Czirják, A. Titilincu, A. Pintea, and Z. Barta. 2009. Carotenoids modulate the effect of coccidian infection on the condition and immune response in moulting house sparrows. J Exp Biol 212:3228-3235.

Pap P.L., C.I. Vágási, G.Á. Czirják, A. Titilincu, A. Pintea, G. Osváth, A. Fülöp, and Z. Barta. 2011. The effect of coccidians on the condition and immune profile of molting house sparrows (Passer domesticus). Auk 128:330-339.

Pap P.L., C.I. Vágási, G. Osváth, C. Mureşan, and Z. Barta. 2010a. Seasonality in the uropygial gland size and feather mite abundance in house sparrows Passer domesticus: natural covariation and an experiment. J Avian Biol 41:653661.

Pap P.L., C.I. Vágási, J. Tökölyi, G.Á. Czirják, and Z. Barta. $2010 b$. Variation in haematological indices and immune function during the annual cycle in the great tit Parus major. Ardea 98: 105-112.
Raja-aho S., M. Kanerva, T. Eeva, E. Lehikoinen, P. Suorsa, K. Gao, D. Vosloo, and M. Nikinmaa. 2012. Seasonal variation in the regulation of redox state and some biotransformation enzyme activities in the barn swallow (Hirundo rustica L.). Physiol Biochem Zool 85:148-158.

Randolph S.E. 1975. Seasonal dynamics of a host-parasite system: Ixodes trianguliceps (Acarina: Ixodidae) and its small mammal hosts. J Anim Ecol 44:425-449.

Rubolini D., G. Colombo, R. Ambrosini, M. Caprioli, M. Clerici, R. Colombo, I. Dalle-Donne, et al. 2012. Sex-related effects of reproduction on biomarkers of oxidative damage in free-living barn swallows (Hirundo rustica). PLoS ONE 7: e48955.

Schaefer H.M., K. McGraw, and C. Catoni. 2008. Birds use fruit colour as honest signal of dietary antioxidant rewards. Funct Ecol 22:303-310.

Sepp T., U. Karu, J.D. Blount, E. Sild, M. Männiste, and P. Hõrak. 2012a. Coccidian infection causes oxidative damage in greenfinches. PLoS ONE 7:e36495.

Sepp T., E. Sild, J. Blount, M. Männiste, U. Karu, and P. Hõrak. $2012 b$. Individual consistency and covariation of measures of oxidative status in greenfinches. Physiol Biochem Zool 85:299-307.

Sepp T., E. Sild, and P. Hõrak. 2010. Hematological condition indexes in greenfinches: effects of captivity and diurnal variation. Physiol Biochem Zool 83:276-282.

Simons M.J.P., A.A. Cohen, and S. Verhulst. 2012. What does carotenoid-dependent coloration tell? plasma carotenoid level signals immunocompetence and oxidative stress state in birds - a meta-analysis. PLoS ONE 7:e43088.

Sorci G. and B. Faivre. 2009. Inflammation and oxidative stress in vertebrate host-parasite systems. Philos Trans R Soc B 364:71-83.

van de Crommenacker J., J. Komdeur, and D.S. Richardson. 2011. Assessing the cost of helping: the roles of body condition and oxidative balance in the Seychelles warbler (Acrocephalus sechellensis). PLoS ONE 6:e26423.

van de Crommenacker J., D.S. Richardson, A.M. Koltz, K. Hutchings, and J. Komdeur. 2012. Parasitic infection and oxidative status are associated and vary with breeding activity in the Seychelles warbler. Proc R Soc B 279:1466-1476.

Versteegh M.A., I. Schwabl, S. Jaquier, and B.I. Tieleman. 2012. Do immunological, endocrine and metabolic traits fall on a single Pace-of-Life axis? covariation and constraints among physiological systems. J Evol Biol 25:1864-1876.

von Schantz T., S. Bensch, M. Grahn, D. Hasselquist, and H. Wittzell. 1999. Good genes, oxidative stress and conditiondependent sexual signals. Proc R Soc B 266:1-12. 
Copyright of Physiological \& Biochemical Zoology is the property of University of Chicago Press and its content may not be copied or emailed to multiple sites or posted to a listserv without the copyright holder's express written permission. However, users may print, download, or email articles for individual use. 